\title{
The Mobile Mapping Van Problem: a matheuristic for capacitated arc routing with soft time windows and depot selection
}

\author{
Pieter Vansteenwegen*, Wouter Souffriau**, \\ Kenneth Sörensen*** \\ *Centre for Industrial Management, Katholieke Universiteit Leuven, Celestijnenlaan 300a, 3001 Leuven, \\ Belgium (Tel: +32-16-322760; e-mail: \{Pieter.Vansteenwegen @ cib.kuleuven.be) \\ **Information Technology, KaHo Sint-Lieven, Gebroeders Desmetstraat 1, 9000 Gent, Belgium (e-mail: \\ Wouter.Souffriau@kahosl.be) \\ *** Faculteit Toegepaste Economische Wetenschappen, Universiteit Antwerpen, Prinsstraat 13, 2000 \\ Antwerpen, Belgium (Kenneth.Sorensen@ua.ac.be)
}

\begin{abstract}
The company Tele Atlas utilises mobile mapping vans to take pictures of streets and road signs. The mobile mapping van problem tries to minimise the number of days required to travel through all streets in a region. Furthermore, hotels need to be selected to spend each night and the position of the sun should be taken into account while driving. This problem is converted into a vehicle routing problem with soft time windows and depot selection. A two step matheuristic (metaheuristic with an embedded linear programming solver) is designed to solve this problem efficiently. Local search moves reduce the number of days and iterated local search, hybridised with a linear solver, reduces the time window violations. The selection of the hotels is updated regularly. High quality results are obtained in a few seconds of computation time for a set of 29 test instances.
\end{abstract}

Keywords: Matheuristic - Vehicle Routing Problem - Depot Selection

\section{INTRODUCTION}

Geographic Information Systems by Google Maps and TomTom, for instance, utilise the digital maps provided by Tele Atlas, the world leading supplier of geographical data. To keep their geographical information up-to-date, "mobile mapping vans" drive around to take pictures of streets and road signs. Afterwards, these pictures are analysed and the information collected is used to update the map database. Tele Atlas wants to minimise the number of days required to travel through all the streets in a certain region. The driver's working hours per day are limited and the driver can stay in any hotel in the region. Selecting a hotel for each night, is a part of the minimisation problem. In this paper, we develop a method to determine the order in which to traverse each street in a certain region and to select the best hotels to do so.

Different street types have to be traversed in different ways. In a one way street, pictures will only be taken in the driving direction. In a narrow enough two way street, pictures can be taken in both directions at the same time. To cover a wide two way street, in which the two driving directions are clearly separated, the van has to drive through the street in both directions. The problem to minimise the total time required to travel through all streets can be modelled as a Mixed Capacitated Arc Routing Problem (MCARP), in which the roads in the network can either be undirected edges (two way streets) or directed arcs (one way streets). Large two way streets can be modelled as two one way streets.

Besides minimising the number of days, Tele Atlas wants to minimise the number of pictures taken towards the sun, since many of these pictures are useless. As a result, many streets can only be successfully traversed during a certain time of the day. This extra objective can be modelled with soft time windows, based on the orientation of the streets. Making pictures towards the sun, i.e. violating the time window, is penalised in the objective function. Note that Tele Atlas does not require vans to completely avoid driving towards the sun. If that was the case, this problem should be modelled with hard time windows.

In order to solve the MCARP with soft time windows efficiently, the problem is first converted to a Vehicle Routing Problem with Soft Time Windows (VRPSTW). Good results can be obtained by solving the MCARP directly, but the technique to convert an undirected CARP into a VRP is well-known and has proven to work well before (Pearn, Assad and Golden 1987; Aminu and Eglese 2006; Baldacci and Maniezzo 2006; Longo, Poggi de Aragão and Uchoa 2006).

The remainder of this paper is organised as follows. In the next section, a short literature review and the problem formulation are presented. Section 3 describes the matheuristic to solve the problem and in Section 4 
experimental results are discussed. Section 5 concludes this paper and adds suggestions for further research.

\section{LITERATURE REVIEW AND PROBLEM FORMULATION}

The problem discussed in this paper is a capacitated arc routing problem with soft time windows and depot selection. Arc routing has recently received a large amount of research interest, but arc routing with time windows is still a relatively underexplored subject. Few metaheuristic optimisation approaches exist. Aminu and Eglese (2006) tackle the Chinese postman problem with time windows using a constraint programming approach. They develop two approaches, one of which tackles the problem directly, whereas the other transforms the problem into a node routing problem. Brandáo and Eglese (2008) develop a tabu search heuristic for the CARP. Reghioui, Prins and Labadi (2007) develop a GRASP method with path relinking for the CARP with time windows. In Belenguer et al. (2006), a memetic algorithm is presented to tackle the MCARP and this is, to the best of our knowledge, currently the only published metaheuristic for the MCARP. Corberán, Mota and Sanchis (2006) intensively compare two different MCARP formulations.

Contrary to arc routing, time windows have been an important object of study in the area of vehicle routing. Undoubtedly the best-studied problem in this area is the Vehicle Routing Problem with (hard) Time Windows (VRPTW), for which a large number of efficient heuristic methods exists (Braysy and Gendreau 2005a, 2005b). The literature on vehicle routing with soft time windows, on the other hand, in which it is allowed to arrive early or late at a customer's location thereby incurring a penalty cost, is much more limited. An early approach to model and solve a multiobjective VRPSTW can be found in (Min 1991), which uses this problem to model the case of a public library distribution system. More recent approaches can be found in (Taillard et al. 1997), who develop a tabu search heuristic and in (Ioannou, Kritikos and Prastacos 2003), who develop a heuristic based on a nearest-neighbour approach. In (Calvete et al. 2007), a goal programming method is developed. One possible way to solve an arc routing problem, also used in this paper, is to transform it into an equivalent node routing problem. The first transformation appeared in (Pearn, Assad, and Golden 1987), more recent and more efficient transformations can be found in (Baldacci and Maniezzo 2006) and (Longo, Poggi de Aragão and Uchoa 2006).

The depot selection, or "hotel selection", is a new and challenging extension of the VRP. To the best of our knowledge, the depots selection, as defined in this paper, has not been treated in the literature. A more detailed literature review about related depot selection problems can be found in (Vansteenwegen, Souffriau and Sörensen 2009).

As mentioned above, our mixed capacitated arc routing problem with soft time windows is first transformed into a vehicle routing problem with soft time windows. This conversion essentially represents each arc and each edge in the original formulation as customers to visit. Details of this conversion can be found in the literature and are beyond the scope of this paper. The objective of the VRPSTW with depot selection is to determine a sequence to visit all streets which minimises the weighted sum of the number of days and the total time window violation. All trips are carried out by a single mapping van and each day, the trip should start at the end hotel of the previous day. At the end of the last day, the van should return to the initial starting hotel of the first day. The working time per day is limited. A time window violation is defined as the time span that the visit starts before the time window opens or ends after the time window closes. In order to determine the total time window violation, each violation is divided by the width of the corresponding time window. The overall objective function is a weighted sum of the number of days $(m)$ and the time window violations ( $w$ is the weight of the violations):

Minimise $\left(m+w * t o t a l \_t i m e \_w i n d o w \_v i o l a t i o n s\right)$

\section{MATHEURISTIC}

An initial solution of the problem is improved in two steps. First, the number of days to visit all locations is minimised, not taking the time windows into account. Selecting the most appropriate hotels during the whole trip plays an important role during this minimisation. Secondly, while keeping the number of days constant, the number of time window violations is reduced by a metaheuristic. To determine and minimise the time window violations in a tour, the metaheuristic is hybridised with an exact linear solver. Then, adding one or more days to the solution is considered, if this significantly reduces the time window violations. These two steps are explained in detail below.

The two-phased approach saves computational effort. In the first phase, in order to evaluate local moves, only a few arcs of the solution need to be considered. This can be computed easily and in constant time. In the second phase, when evaluating the change in time window violations after a certain move, the possible time window violation needs to be computed again for each visit of the tour under consideration, requiring substantial computation time. The total computation time of the algorithm would increase drastically if this expensive evaluation would also be required when moves are considered to reduce the number of days.

\subsection{Initialisation}

During the initialisation, no time windows are taken into account. An initial solution is generated based on the "nearest neighbour" principle. At every iteration, the point closest to the previous point in the tour is added. A point can only be added to the tour, if one of the hotels can be reached (within the allowed time). If no more visits can be added to the current tour, the tour is closed with the nearest hotel. This hotel will also be the starting point of the next tour.

The first tour starts at the initial starting point. The last tour must end again at the initial starting point. If this is not feasible with the tour under construction, one extra tour, without visits, is added to reach the initial starting point. 
To speed up the computation process, the nearest hotel is precomputed for each point. This list of "nearest hotels" will be useful during different steps of the solution procedure.

\subsection{Minimise the number of days}

In order to minimise the number of days, two well known local search moves, minimising the travel time in each day, are combined with a procedure to improve the hotel selection. Next, a procedure called "Reduce" is applied that tries to eliminate one day from the schedule by moving each visit of a given day to one of the other days. Since the end hotel of one day must be the starting hotel of the next day, the remaining days also need to be relinked, when a day is removed. If the removal appears feasible, the travel time in the remaining days is minimised and Reduce is applied again.

The first local search move to reduce the travel time, "TwoOpt" (Lin 1965), removes two edges from the tour to replace them with two new edges not previously included in the tour. The tour has to remain closed and the total travel time should be reduced. The second local search move, "Relocate" (Savelsbergh 1992), moves a visit from one day to another, if this reduces the total travel time. These neighbourhoods are sequentially explored in a best improving fashion.

Next, "ImproveHotels" tries to replace one or more of the current hotels by another hotel in order to reduce the total travel time. ImproveHotels considers every set of two consecutive tours. The start hotel of the first tour and the end hotel of the second tour remain fixed, due to the other tours in the solution. While the "intermediate hotel", the end hotel of the first tour (and the starting hotel of the second tour), is considered for replacement. For every visit of these two tours, its nearest hotel is added to a list of possible intermediate hotels. One by one, these hotels will be considered as the new intermediate hotel. First, all visits included in these tours are temporarily removed from the solution. Then, all visits are inserted iteratively, at the position that requires the least extra travel time, taking into account the visits that were inserted before and the intermediate hotel under consideration. If all visits can be inserted in this way, the intermediate hotel is considered suitable. The intermediate hotel resulting in the lowest total travel time, is selected. The contribution of "ImproveHotels" in obtaining high quality solutions is illustrated in the experimental results section.

Only straightforward heuristics are implemented since a small amount of computation time should be spent to find the absolute minimal number of days to visit all locations (without taking time windows into account). It can be expected that one or more days will be added anyway to this minimum in order to obtain an acceptable amount of time window violations in the next step.

\subsection{Minimise the time window violations}

In order to minimise the time window violations, while keeping the number of days constant, Iterated Local Search (ILS) (Lourenço, Martin and Stützle 2003) is applied. ILS is a metaheuristic based on a simple idea: iteratively build sequences of solutions that are generated by an embedded local search heuristic. This leads to much better solutions than repeating random trials of the same heuristic. ILS has shown before to work well on problems with time windows (Ibaraki et al. 2005; Hashimoto, Yagiura and Ibaraki 2008; Vansteenwegen 2008).

Starting from a solution with a given number of days and a minimised travel time per day, the time window violations are minimised without changing the number of days. As a local search heuristic in the ILS framework, "ReOpt" is applied. ReOpt relocates a chain of consecutive visits inside a day or from one day to another. In this way, ReOpt generalises "Or-Opt" (Or 1976), relocating a chain within a day, and Relocate (Savelsbergh 1992), moving a visit from one day to another. A move is only considered if the time window violations can be reduced. Using a heuristic procedure, we determine relatively fast, whether a certain move will reduce the violations and what the minimal violation reduction will be. Some opportunities for reducing the time window violations are not considered in this quick evaluation. Only the minimal guaranteed reduction is calculated. The move with the highest minimal reduction is selected for execution. During execution of the move, a straightforward Linear Programming (LP) problem (Figure 1) is solved to obtain the minimal possible time window violations, for the new sequence of visits. This LP determines the optimal starting time for each visit without changing the order of the visits in the sequence, potentially allowing the van to wait before driving through a street. It is solved by an embedded linear programming solver. The reduction in violations will always be at least as high as the minimal reduction guaranteed by the fast evaluation, and often even higher. It would be too time consuming to solve the LP during the evaluation of every possible move, therefore, the fast evaluation is implemented instead.

$$
\begin{aligned}
& \text { Minimise violations }=\sum_{i=1}^{n}\left(\frac{\text { overtime }_{i}}{\text { close }_{i}-\text { open }_{i}}+\frac{\text { undertime }_{i}}{\text { close }_{i}-\text { open }_{i}}\right) \\
& \text { s.t. } \\
& \text { arrive }_{i}=\text { begin }_{i-1}+\text { duration }_{i-1}+\text { distance }_{(i-1, i) ;} ; \quad i=1, \ldots, n+1 \\
& \text { begin }_{i}>=\text { arrive }_{i} \text {; } \\
& \text { begin }_{i}>=\text { open }_{i}-\text { undertime }_{i} \text {; } \\
& \text { arrive }_{0}=0 \text {; } \text { begin }_{n+1}=\text { close }_{i} \text {; } \\
& i=0, \ldots, n+1 \\
& i=0, \ldots, n+1 \\
& i=0, \ldots, n
\end{aligned}
$$

Fig. 1. LP to minimise the overall time window violations.

During ILS, when a local optimum is reached, the solution will be shaken in order to reach another, and hopefully better, local optimum. During this perturbation phase, a number of visits will be removed in each tour. "Perturbation" uses two parameters: NumberToRemove indicates how many consecutive visits to remove in each tour, while StartPosition indicates the position in each tour to start the removal process. If, during the removal, the end location is reached, the removal continues after the start location. After the removal, all visits following the removed visits are shifted to the beginning of the tour as much as possible, in order to maximise the opportunity for other visits to be included. Next, to perturb the local optimum even more, ImproveHotels 
is applied once before and once after all the removed visits are inserted again. "Insertion" is carried out based on two criteria. First, the best insertion position with respect to extra travel time is determined for each unvisited location. Secondly, the location with the highest value of "travel time from the initial start hotel" minus "extra travel time required for insertion" is selected for insertion. These criteria are based on the insertion heuristic "II" of Solomon (1987). If it is impossible to insert all visits this way, the perturbation phase is first reversed, i.e. the removed visits are inserted again in their original sequence, and then applied again after updating the parameters. Again, no time windows are taken into account during ImproveHotels or Insertion.

As acceptance criterion for ILS, "random walk" is used (Lourenço, Martin and Stützle 2003). The heuristic always continues the search from the current solution, it never returns to the current best solution to continue. Of course, the best found solution is always retained. This random walk acceptance criterion enhances the diversification.

The ILS heuristic loops until, during a fixed number of iterations, no improvements are identified for the best solution determined so far. After the perturbation phase, ReOpt is applied until a local optimum is reached. If this solution is better than the incumbent solution, the solution is recorded and NumberToRemove is reset to one for the next perturbation phase. After each acceptance phase, StartPosition is increased by the value NumberToRemove and NumberToRemove is increased by one for the next perturbation phase. If NumberToRemove equals $n /(3 * m)$, it is reset to 1 ( $n$ the number of locations; $m$ the number of days). By using the perturbation parameters as described above, different visits are removed during every perturbation phase and during the entire procedure, most likely, every visit is removed at least once. In this way, the entire solution space is better explored.

The maximum number of locations to remove $\left(n /\left(3^{*} m\right)\right)$ and the maximum number of iterations without improvement $(n / m)$ are the only parameters to predetermine in this heuristic. It appears that further increasing the maximum number of iterations without improvement does not significantly improve the results and only increases computation times. The difficulty of the problem, and therefore the required number of iterations, depends on the average visits per day $(=n / m)$.

After minimising the time window violations for this fixed number of days, an extra day is added to the solution in order to increase the possibility to further reduce the time window violations. Again the ILS heuristic is applied to minimise the violations for this number of days. If the overall objective, i.e. the weighted sum of the number of days and time window violations, is reduced by adding one day to the initial solution, another day is added and the problem is solved again.
Figure 2 presents the pseudo code of the complete matheuristic.

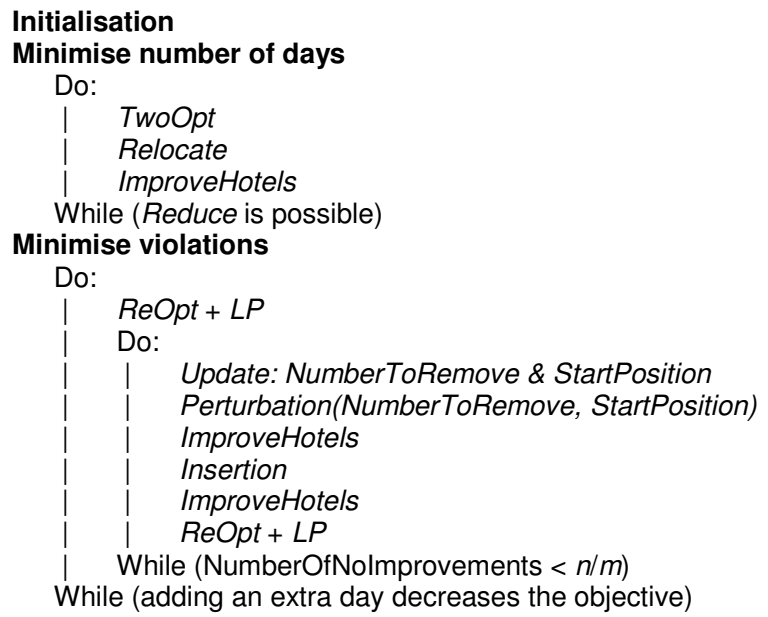

Fig. 2. Pseudo code of the matheuristic.

\section{EXPERIMENTAL RESULTS}

In the literature, no test sets are available for the VRP with soft time windows and depot selection. Therefore, a new set of problems is constructed based on the well-known test set of Solomon (1987) $(n=100)$. Three extra depots (hotels), next to the initial starting point, are added to these instances. For every problem, an extra depot is available at the coordinates of point 1,11 , and 21 . The opening hours of all the depots are equal to the opening hours of the initial starting point. The data sets are available from the authors upon request. During the experimental tests, the weight of the time window violations $(w)$ is set to 0.1 . These problems are too complex to be solved to optimality by a commercial solver in reasonable time. Therefore, two results to compare with are published as well: the overall objective obtained by our algorithm when no extra hotels are available and the overall objective of the initial solution (before reducing the time window violations). All computations were executed on a personal computer Intel Core 2 with $2.50 \mathrm{GHz}$ processor and 3.45 GB Ram.

Table 1 presents the results for the new set of instances. Column one gives the instance's name. As a reference, column two states the overall objective when no extra hotels are available and in column three the overall objective of the initial solution is mentioned. In column four, the overall objective of the matheuristic solution is presented. The computation time in the last column is expressed in seconds.

On average, the computation time for these problems is only $1,6 \mathrm{~s}$ and the overall objective is reduced with $38 \%$ compared to the initial solution. Compared to the situation where no extra hotels are available, the objective is reduced with $11 \%$. This indicates the benefit of the hotel selection. For seven out of nine Solomon problems with clustered points, c101-c109, the best found solutions are the ones with no extra hotels. This can be explained by the fact that the points are clustered in such a way that every node in a cluster can be visited, starting and ending in the initial starting point. 
It should be noticed that real-world problems have much larger dimensions. For instance, the bicycle network in one of the ten Belgian provinces consists of around 2800 edges $(n)$ for a total length of $6800 \mathrm{~km}$. With an average speed of $30 \mathrm{~km}$ per hour (while taking pictures) and working 8 hours per day, this planning problem requires at least 30 days $(\mathrm{m})$ for one van with an average of 93 nodes per day. Especially the high number of nodes per day will significantly slow down the LP solver to optimise each route. Therefore, the calculation time for the benchmark instances from the literature should be very small, since real-world instances will require many times more computational effort.

Table 1. Results for the new test instances $(n=100)$

\begin{tabular}{|c|c|c|c|c|}
\hline Name & $\begin{array}{c}\text { No extra } \\
\text { hotels }\end{array}$ & $\begin{array}{c}\text { Initial } \\
\text { solution, } \\
\text { three extra } \\
\text { hotels }\end{array}$ & $\begin{array}{l}\text { matheuristic } \\
\text { solution, three } \\
\text { extra hotels }\end{array}$ & $\mathrm{CPU}(\mathrm{s})$ \\
\hline c101 & 10.27 & 21.01 & 10.69 & 4 \\
\hline c102 & 9.81 & 19.82 & 10.69 & 3 \\
\hline c103 & 9.49 & 17.51 & 10.35 & 1 \\
\hline c104 & 9.03 & 14.95 & 9.22 & 2 \\
\hline c105 & 10.36 & 17.02 & 10.04 & 3 \\
\hline c106 & 10.09 & 15.69 & 9.69 & 3 \\
\hline c107 & 9.37 & 14.40 & 9.72 & 3 \\
\hline c108 & 9.42 & 12.56 & 9.53 & 3 \\
\hline c109 & 9.10 & 10.96 & 9.31 & 2 \\
\hline r101 & 17.59 & 37.13 & 12.47 & 2 \\
\hline r102 & 16.03 & 30.44 & 12.33 & 1 \\
\hline r103 & 14.31 & 24.24 & 11.22 & 1 \\
\hline r104 & 12.13 & 16.19 & 9.01 & 1 \\
\hline r105 & 12.93 & 20.49 & 10.43 & 2 \\
\hline r106 & 12.48 & 17.69 & 10.39 & 1 \\
\hline r107 & 11.94 & 15.25 & 9.93 & 1 \\
\hline r108 & 10.32 & 11.95 & 9.01 & 1 \\
\hline r109 & 11.29 & 13.86 & 9.59 & 1 \\
\hline r110 & 11.13 & 12.58 & 9.38 & 1 \\
\hline r111 & 11.08 & 13.77 & 9.40 & 1 \\
\hline r112 & 9.31 & 10.02 & 9.12 & 1 \\
\hline rc101 & 12.90 & 20.93 & 9.82 & 2 \\
\hline $\mathrm{rc} 102$ & 11.83 & 18.50 & 9.60 & 1 \\
\hline $\mathrm{rc} 103$ & 12.01 & 15.68 & 9.29 & 1 \\
\hline rc104 & 10.22 & 12.67 & 9.29 & 1 \\
\hline rc105 & 13.50 & 22.05 & 9.82 & 1 \\
\hline rc106 & 10.68 & 13.83 & 9.34 & 1 \\
\hline rc107 & 10.29 & 11.89 & 9.27 & 1 \\
\hline $\mathrm{rc} 108$ & 9.59 & 10.60 & 9.11 & 1 \\
\hline average & 11.33 & 17.02 & 9.90 & 1.6 \\
\hline
\end{tabular}

Table 2 summarises different experimental results: no extra hotels (column one), initial solution (column two), matheuristic solution (column three) and when ImproveHotels is not applied (column four). Row one states, for these algorithms, the average overall objective and row two gives the average number of tours. In row three the average violation is mentioned an in row four, the average travel time is presented.
These results clearly indicate that the matheuristic obtains high quality results considering the overall objective function, the number of tours and the violation, as well as the travel time. If ImproveHotels is not implemented to update the hotel selection regularly, on average, one extra day is required for each instance, the overall objective is increased with $17 \%$ and the total travel time is increased with $6 \%$. This clearly illustrates the contribution of ImproveHotels in obtaining high quality results. Finally, these results illustrate that the initialisation does a good job in minimising the travel time, but it does not take the time window violations into account.

Table 2. Summary of experimental results

\begin{tabular}{|c|c|c|c|c|}
\hline & $\begin{array}{c}\text { No } \\
\text { extra } \\
\text { hotels }\end{array}$ & $\begin{array}{c}\text { Initial } \\
\text { solution, } \\
\text { three extra } \\
\text { hotels }\end{array}$ & $\begin{array}{c}\text { Matheuris- } \\
\text { tic solution, } \\
\text { three extra } \\
\text { hotels }\end{array}$ & $\begin{array}{c}\text { No } \\
\text { Improve } \\
\text {-Hotels }\end{array}$ \\
\hline $\begin{array}{r}\text { Average overall } \\
\text { objective }\end{array}$ & 11.33 & 17.02 & 9.90 & 11.57 \\
\hline $\begin{array}{r}\text { Average number } \\
\text { of tours }\end{array}$ & 9.93 & 9.00 & 9.00 & 10.00 \\
\hline Average violation & 14.31 & 80.25 & 9.98 & 15.73 \\
\hline $\begin{array}{r}\text { Average travel } \\
\text { time }\end{array}$ & 4667 & 4594 & 4434 & 4694 \\
\hline
\end{tabular}

\section{CONCLUSIONS AND POSSIBLE EXTENSIONS}

The mobile mapping van problem, selecting the most appropriate hotels to spend the night, optimising the route of the vans, and taking into account the position of the sun, can be converted to a vehicle routing problem with soft time windows and depot selection. High quality solutions are obtained in only a few seconds of computation time by hybridising an ILS metaheuristic with a linear programming solver and updating the hotel selection. Experimental results on a new set of 29 test instances indicate the strength of the algorithm.

The test set is based on a set of instances from the literature and the instances can be used in the future as a benchmark for this type of problem. The limited computation time for these relatively small instances offers a lot of opportunities for applying this algorithm to larger real-world instances. Furthermore, during Relocate and ReOpt, the number of possible destinations to consider for a certain visit, can be limited based on the distance to other visits or based on the time windows of other visits.

In order to deal with real-life instances, more attention should go to efficiently converting the mixed capacitated arc routing problem to a vehicle routing problem or solving the problem directly as an arc routing problem. 


\section{REFERENCES}

Aminu, U. and Eglese, R. (2006). A constraint programming approach to the Chinese postman problem with time windows. Computers \& Operations Research, 33(12), 3423-3431.

Baldacci, R. and Maniezzo, V. (2006). Exact methods based on node-routing formulations for undirected arc-routing problems. Networks, 47, 52-60.

Belenguer, J.M., Benavent, E., Lacomme, P., and Prins, C. (2006). Lower and upper bounds for the mixed capacitated arc routing problem. Computers \& Operations Research, 33(12), 3363-3383.

Brandáo, J. and Eglese, R. (2008). A deterministic tabu search algorithm for the capacitated arc routing problem. Computers \& Operations Research, 35 (4), 1112-1126.

Braysy, O. and Gendreau, M. (2005a). Vehicle routing problem with time windows, part I: route construction and local search algorithms. Transportation Science, 39 (1), 104-118.

Braysy, O. and Gendreau, M. (2005b). Vehicle routing problem with time windows, part II: metaheuristics. Transportation Science, 39 (1), 119-139.

Calvete, H., Galé, C., Oliveros, M.-J., and Sánchez-Valverde, B. (2007). A goal programming approach to vehicle routing problems with soft time windows. European Journal of Operational Research, 177 (3), 1720-1733.

Corberán, A., Mota, E., and Sanchis, J. (2006). A comparison of two different formulations for arc routing problems on mixed graphs. Computers \& Operations Research, 33(12), 3384-3402.

Hashimoto, H., Yagiura, M., and Ibaraki, T. (2008). An iterated local search algorithm for the time-dependent vehicle routing problem with time windows. Discrete Optimization, 5, 434-456.

Ibaraki, T., Imahori, S., Kubo, M., Masuda, T., Uno, T., and Yagiura, M. (2005). Effective local search algorithms for routing and scheduling with general time-window constraints. Transportation Science, 39(2), 206-232.

Ioannou, G., Kritikos, M., and Prastacos, G. (2003). A problem generator-solver heuristic for vehicle routing with soft time windows. Omega, 31(1), 41-53.

Lin, S. (1965). Computer solutions of the traveling salesman problem. Bell System Technical Journal, 44, 2245-2269.

Longo, H., Poggi de Aragão, M., and Uchoa, E. (2006). Solving capacitated arc routing problems using a transformation to the CVRP. Computers \& Operations Research, 33 (6), 1823-1837.

Lourenço, H., Martin, O., and Stützle, T. (2003). Iterated local search. In Glover, F. and Kochenberger, G. (ed.), Handbook of Metaheuristics, 321-353. Kluwer, Boston.

Min, H. (1991). A multi-objective vehicle routing problem with soft time windows: the case of a public library distribution system. Socio-Economic Planning Sciences, 25 (3), 179-188.

Or, I. (1976). Traveling salesman-type combinatorial problems and their relation to the logistics of regional blood banking. Ph.D. thesis, Northwestern University, Evanston, IL.
Pearn, W.-L., Assad, A., and Golden, B. (1987). Transforming arc routing into node routing problems. Computers \& Operations Research, 14 (4), 285-288.

Reghioui, M., Prins, C., and Labadi, N. (2007). GRASP with path relinking for the capacitated arc routing problem with time windows. EvoWorkshops 2007, LNCS 4448, Springer, 722-731.

Savelsbergh, M. (1992). The vehicle routing problem with time windows: minimizing route duration. Journal of Computing, 4, 146-154.

Solomon, M. (1987). Algorithms for the Vehicle Routing and Scheduling Problems with Time Window Constraints. Operations Research, 35(2), 254-265.

Taillard, E., Badeau, P., Gendreau, M., Guertin, F., and Potvin, J.-Y. (1997). A tabu search heuristic for the vehicle routing problem with soft time windows. Transportation science, 31(2), 170-186.

Vansteenwegen, P. (2008). Planning in tourism and public transportation - Attraction selection by means of a personalised electronic tourist guide and train transfer scheduling. Ph.D. thesis, Katholieke Universiteit Leuven, Belgium.

Vansteenwegen, P., Souffriau, W., and Sörensen, K. (2009). The traveling salesperson problem with hotel selection. Submitted to Journal of the Operational research Society.

\section{ACKNOWLEDGEMENTS}

Pieter Vansteenwegen is a post-doctoral research fellow of the "Fonds Wetenschappelijk Onderzoek - Vlaanderen (FWO)". 